\title{
Social Network Analysis of Public Opinions Dissemination on Mobile Internet
}

\author{
Cui Yaqun, Cao Haiqing, Qiu Yue, Yao Cuiyou* \\ Information School, Capital University of Economics and Business, \\ Beijing, China \\ Email: ycy@cueb.edu.cn
}

\begin{abstract}
We took "Wei Zexi Event" as a case to study the communication mechanism of mobile Internet public opinion. Then we got the public opinion data of mobile terminal through Sina microblog, and generated network structure diagram with Gephi software. We analyzed the characteristics of both the network structure and the transmission process of network public opinion. Finally, we put forward suggestions to control public opinion of mobile internet with public opinion monitor and response methods and hoped to improve laws and regulations system.
\end{abstract}

Keywords-Mobile Internet; Social Network Analysis; Public Opinion Dissemination

\section{INTRODUCTION}

According to the 38th China Internet Development Statistics Report released by China Internet Network Information Center, the number of mobile phone users has added to 656 million in China before June, 2016. Mobile network has become an important channel for the public to express their own demands on public events. With the rapid spread of intelligent mobile terminals, more and more internet users have been used to participate in the discussion of public events with mobile terminals. At the same time, because of the asymmetry of public opinion information and the rapid spread of information under the mobile Internet, rumors in the network are growing and even threatening social stability. The rapid development of mobile Internet gives the evolution and generation of internet public opinion new features. And new challenges are put forward to deal with network public opinion. Therefore, the research on public opinion dissemination and guidance under the mobile Internet has become a new hotspot.

The research on public opinion in mobile internet mainly focuses on the evolution of public opinion, the mechanism of formation and the control strategies of public opinion. Many researchers have gained some results on the evolution and dissemination of public opinion in the mobile environment. Hu Lili et al. studied the particularity of the internet public opinion and its social influence in the mobile Internet [1]. Shang Yuefeng studied the transmission characteristics of network public opinion at all stages of mobile internet through the analysis of public opinion cases [2]. Wang Xiwei et al. studied the propagation path and its law of network public opinion information in the mobile environment by comparing the dissemination of the hotspot topic on the mobile and nonmobile sides [3]. Xiong Yin et al. focused on the characteristics of the dissemination of internet public opinion and the existing risk in WeChat platform [4]. Wang Yuzhu mainly contrasted the microblog to WeChat platform's role and impact in the dissemination process of public opinion [5].

Now some scholars use social network analysis software to gain dissemination network of public opinions, analyze the indicators of social network, and identify key nodes to reveal the features of internet opinions. Wang Xiwei et al. contrasted the differences in the dissemination of public opinion information between mobile and nonmobile through social network analysis [6]. Weng Shihong et al. used the social network analysis method to analyze the network structure characteristic of microblog public opinion interconnection in public agenda setting, and put forward the behavior logic of internet public opinion interaction in public agenda setting [7]

In summary, the current research lacks the study about network public opinion propagation in mobile Internet by social network analysis method. In the paper, the social network analysis method is used in public opinion under mobile internet. The public opinion data of the mobile terminals can be got from Sina microblog. The characteristics of public opinions under mobile internet are analyzed through contrasting the social network index of different public opinion stage by social network analysis method. For clarity, the paper is based on the analysis of "Wei Zexi Event".

\section{DATA SOURCE}

During the May Day holiday in 2016, the "Wei Zexi Event" aroused the public to pay more attentions. This event initially get a hot topic on the Zhihu platform. Subsequently, micro-blog, WeChat and other social platforms continue to ferment. A college student named Wei Zexi died by suffering from synovial sarcoma on April 12, 2016. A post with a title "How is Wei Zexi?" is published in Zhihu platform, in which his father with Zexi's account told internet users that Wei Zexi has been dead, and which caused internet users' attention. Since May 1, files with titles of "the death of young Wei Zexi", "a young man died in the hands of Baidu and the army hospital" were published, which were the most widely disseminated in microblog, $\mathrm{WeChat}$ and public opinion is into the outbreak stage. Then there were some posts saying that Wei Zexi's medical experiences brought public opinion hot topics. At the same time, the mainstream 
media also reported on the incident. After the relevant regulatory authorities began to investigate Baidu and the related hospitals and reported the survey results on May 9. Then the public opinion begin to gradually decline. This paper gets the event data from Sina Microblog by internet mobile terminal and analysize these data. Wei Zexi incident heat is shown in Figure 1 by micro-blog data center from April 28 to May 25.

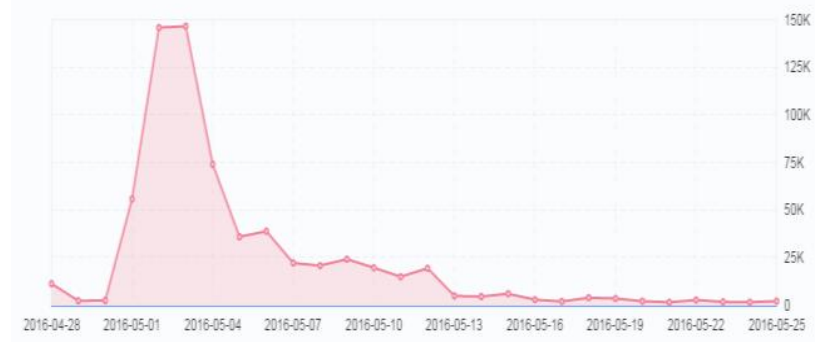

Fig. 1 Data related to "Wei Zexi Event" from mobile terminals in Sina microblog.(From Sina microblogging data center)

Obviously the attention changing of "Wei Zexi Event" from mobile terminals is shown in Figure 1. From figure 1 the attention of public opinion from mobile terminals can be divided into four public opinion evolution stages including latency period(4.28-4.30), outbreak period (5.15.6), spread period (5.7-5.12) and recession period (5.135.25).In the 28 days selected, the times of attention is more than 10,000 in 13 days and more than 30,000 in 6 days. Especially the times of attention from mobile terminals are more than 140,000 on May 2 and May 3.

\section{ANALYSIS METHOD}

\section{A. Research Ideas}

In this paper, social network analysis method is used to study "Wei Zexi Event". Internet users are used as nodes, comments and forward relationship between internet users are as the edge. First the event data are collected by Sina microblog. Then the data from the mobile terminals are filtered out. The relationship matrix of the internet users is established in different public opinion development stage. Gephi software is used to map the social network of the dissemination about public opinion in different stages. Finally, the transmission characteristics of the internet public opinion in the mobile environment is analysized through the comparison of the network density, degree center and average path length of the social network graphs. Controlling strategies about public opinion are put forward. Network density can be used to represent the degree of intimate interaction between nodes. The greater network density indicates, the more interaction and the more intimate relationship between nodes. The centrality degree can be used to identify the key nodes in the relational network. The greater degree of a node is, the greater power of the node is. The node can be regarded as the opinion leader. The average path length is the average of the distance between all nodes that describes the degree of separation between nodes. It can be used to measure the efficiency of public opinion dissemination. The smaller average path length is, the faster the public opinion disseminates.

\section{B. Mobile Data Acquisition and Processing}

Sina microblog as a data source, data collection and processing ideas are as follows:

- Taking "Wei Zexi" as the key word, microblog keyword data are collected by reptile software from April 28, 2016 to May 25, 2016, including bloggers name, blog post independent website, blog posting time, releasing terminal, forwarding number, comments and other basic information.

- Sort the keyword data according to the number of blog forwarding, and remove the non-mobile data. Then select the first three nodes with the largest number of blog posts in each phase as primary node. Their corresponding posts are taken as first class post.

- Collect the blog post comments forwarding data according to the primary node post independent site to, namely on the post second, third, and fourth level data of forwarding comments. In this step, mainly collect popular blog post and popular commentary data. If there is a popular forwarding and popular comment in a blog post, the next level's forwarding and comments data will be collected, otherwise select one of the data from the mobile side in its forwarding and comment data and no longer acquire the next level of data. The data collected in this step mainly includes the blogger name, the blog independent website, and the blog posting time.

- Use Excel software to sort out the collected data, 247 nodes were collected. Then put relevant data that the post release time belong to the same stage of public opinion in the same form, and establish the relationship matrix of different phase of public opinion according to the forwarding and comment relationship between the nodes. The relationship matrix (part) is shown in Table 1.

TABLE I. RELATIONSHIP MATRIX (PARTIAL) OF RECESSION PERIOD

\begin{tabular}{|c|c|}
\hline Source & Target \\
\hline 68 & 1 \\
\hline 3 & 2 \\
\hline 64 & 4 \\
\hline
\end{tabular}

\section{RESULTS ANALYSIS AND DISCUSSION}

This paper uses Gephi software to analyze the process of public opinion transmission about "Wei Zexi Event". This software has a strong visualization and dynamic analysis capabilities. We can use this software to draw the overall relationship network of the mobile public opinion dissemination(in Figure 2) and the social network of different public opinion stages (in Figure 3 - 6), and compare social network measurement indicators of the different stages to analyze the public opinion dissemination process. 
A. Analysis of the General Relationship Diagram of Public Opinion

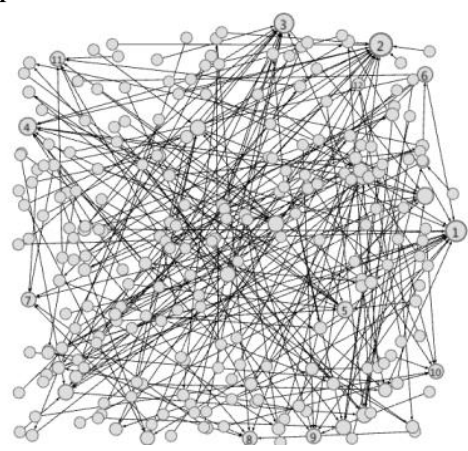

Fig. 2 Public opinion dissemination's social network map (April 28 May 25).

In Figure 2, only a few nodes are labeled and larger in shape, most of the nodes are not labeled and smaller in shape. That is, the degree distribution with the power law distribution indicates that the total relation graph is a scalefree network. In this type of social network, a few nodes on the overall structure of the network will have a greater impact on the total network structure. In which the label of the nodes that have larger degree respectively is 2(degree: 18), 1(degree: 15) and 3(degree: 13). These nodes can be regarded as the opinion leaders in the internet public opinion dissemination. The network density of the Figure 2 is 0.004 , which indicates that the links between the nodes are not close and less interactive. There is only one-way forward and comment between internet users. The average path length of Figure 2 is 2.427 , which indicates that the nodes are easier to establish contact and there is a small world phenomenon in the network. So the public opinion disseminate quickly, the relevant departments should take effective measures to guide the development of public opinion in a timely manner.

\section{B. Contrastive Analysis of Different Public Opinion Stage}

The evolution of public opinion at all stages of the social network map is shown in Figure 3, 4, 5 and 6. Obviously there are more nodes in the latency and outbreak periods. Fewer nodes in the spread period and the recession period are obviously reduced. There are 69 nodes in the latency period, 131 nodes in the outbreak period. That means the latency period has a greater impact on the evolution of public opinion, especially in outbreak stage. In the latent stage of public opinion, the relevant regulatory authorities and companies involved in public opinion events should respond to the incident in time and take a stand, so as to reduce the slow response which can make internet users dissatisfied and even produce rumors. There are 22 nodes in the spread period, which are 109 less than the nodes in the outbreak period. This change shows that the outbreak of public opinion is a critical period of controlling public opinion. In this stage, regulatory departments should investigate, clarify the event and reply to internet users as soon as possible. Meanwhile the major media should also report the fact to the public and prohibit rumors. The social network indicators of each public opinion stage are shown in Table 2 - Table 3.

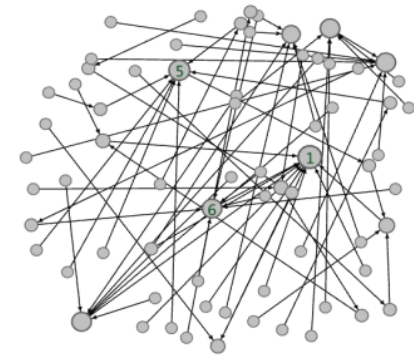

Fig. 3 Social network map of the latency period (April 28 - April 30).

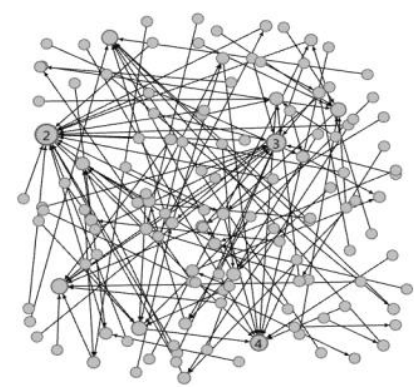

Fig. 4 Social network map of the outbreak period (May 1 - May 6).

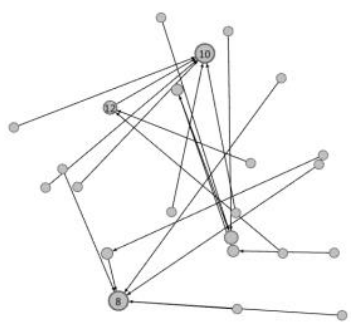

Fig. 5 Social network of the spread period (May 7 - May 12).

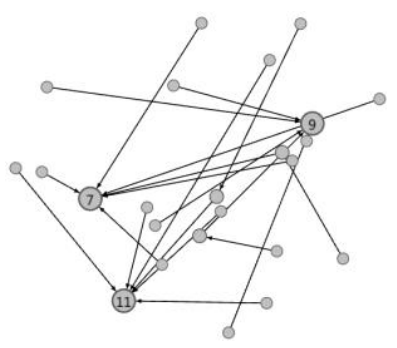

Fig. 6 Social network of the recession period (May 13 - May 25).

TABLE II. NETWORK DENSITY AND AVERAGE PATH LENGTH OF SOCIAL NETWORK GRAPHS AT EACH STAGE

\begin{tabular}{|c|c|c|}
\hline Period & Density & Average path length \\
\hline Latency & 0.014 & 1.903 \\
\hline Outbreak & 0.007 & 1.856 \\
\hline Spread & 0.04 & 1.231 \\
\hline Recession & 0.038 & 1.125 \\
\hline
\end{tabular}

TABLE III. DEGREE CENTRALITY OF THE TOP THREE NODES

\begin{tabular}{|c|c|c|c|}
\hline Latency & Outbreak & Spread & Recession \\
\hline 1 & 2 & 8 & 9 \\
\hline 5 & 3 & 10 & 11 \\
\hline 6 & 4 & 12 & 7 \\
\hline
\end{tabular}


From Table 2, we can draw a conclusion that if the network density of each stage is smaller, the average path length is shorter. Smaller network density indicates less interaction between nodes, no intimate relationship between nodes is established, the smallest network density at outbreak stage is 0.007 , which means interaction of internet users is less, which also reflects in the outbreak period of public opinion, more and more mobile users are more inclined to express their own views on public opinion, and not to interact to other Internet users, and which is caused by the rapid development of mobile internet. Internet users can express their views on an event by mobile terminals at whenever and wherever, and browse other internet users' views which have an impact on the above internet users. The average path length is relatively short and no more than 2, which indicates that each node can communicate with other nodes with help of not up to 2 nodes, the public opinions disseminate quickly, and there is good accessibility between nodes. Taking into account the small network density in Table 2, it reflects the lack of interaction between the nodes, which indicates that mobile users are more inclined to forward a blog to express a certain view of the identity, rather than have more views of the collision. Obviously in the process of public opinion dissemination there is a "silent spiral" phenomenon.

The degree centrality of the nodes in Table 3 is the largest of the three nodes in the outbreak period, and the degree centrality of the other nodes is relatively small. It shows that the three nodes in the outbreak period are at the center of the public opinion dissemination network and have great influence on other nodes, which can be regarded as the key nodes in the process of public opinion dissemination. Relevant regulators can guide public opinion by cultivating or establishing opinion leaders, especially in the outbreak of public opinion, in order to reduce the negative social impact caused by rumors.

\section{CONCLUSIONS}

In the paper the degree centrality of nodes, the network density and average path length of the relational network are used to identify the key nodes in public opinion dissemination. We find that the interaction between the nodes in the mobile internet environment is less, but the dissemination efficiency is faster. We also find that mobile users are more inclined to express their views on an event through the one-way rather than multi-direction. Meanwhile opinion leaders in the mobile internet environment have a great impact on the dissemination of internet public opinion and the speed and scope of dissemination. In addition, through the crawling data we find that the recession period of mobile public opinion takes a longer time. That is because the mobile internet users can more easily use the fragmented time to focus on public opinion events.

To ease the heat of public opinion, relevant departments should take a positive response to the discussion hot spots of internet users to reduce the negative impact of rumors. At the same time they also need to cooperate with the opinion leaders to guide public opinion. In the spread and recession period of public opinion, they should take action to monitor the public opinions to deal with possible derivative public opinion. In addition, they should make appropriate laws and regulations to punish internet users who maliciously create and disseminate rumors to create a healthy network environment.

\section{ACKNOWLEDGMENT}

This research was financially supported by National Social Science Fund (Project No. 15AGL001).

\section{REFERENCES}

[1] H. Lili, and C. Aiting, "Research on Network Public Opinion in Mobile Internet- Taking the girls' attendance incident as an example," Journal of News Research, vol. 7, May. 2016, pp. 125.

[2] S. Yuefeng, "The Development of Network Public Opinion in Mobile Internet," News Dissemination, vol. 6, Jun. 2016, pp. 26-27.

[3] W. Xiwei, X. Yunfei, Z. Dan, and Z. Jun, "Research on the Information Dissemination Paths and Dissemination Law of Online Public Opinion Under the Mobile Environment," Information Studies: Theory \& Application, vol. 39, Sept. 2016, pp. 107-112.

[4] X. Yin, and Z. Zhenyu, "The Dissemination Characteristics and the Risk of Public Opinion of WeChat," Modern Communication, vol. 2, Feb. 2016, pp. 79-82.

[5] W. Yuzhu, "Symbiosis and Change of microblog and WeChat in Public Opinion Events: A Case Study of 'Dongguan Sweating,,' XIN WEN JIE, vol. 11, Nov. 2015, pp. 59-63.

[6] W. Xiwei, X. Yunfei, Z. Dan, and L Jiaxing, "The Study of Network Public Opinion Dissemination with Social Network Analysis Under the Mobile Environment: A Case of 'Haze' in Sina Micro-blog," Library and Information Service, vol. 59, Apr. 2015, pp. 14-22.

[7] W. Shihong, and Z. Yun, "Social Network Analysis on Micro-blog Public Opinion Diffusion in Public Agenda," WUHAN UNIVERSITY JOURNAL (Humanity Sciences), vol. 69, Jan. 2016, pp. 109-118. 